\title{
Print and publishing and the future of Augmented Reality
}

\author{
Christine Perey* \\ PEREY Research \& Consulting, Montreux, Switzerland \\ http://www.perey.com
}

\section{Summary}

For Augmented Reality to reach beyond novelty (the simple "wow" factor) and become utilitarian and enjoyable regardless of where the user works or plays, there will need to be:

- a nearly infinite amount of content, just as there is today on other digital platforms (e.g., the Web),

- an easy system for the owners of content to build or find complementary digital data and have their content be viewed and associated with additional digital information in AR-assisted applications, and

- many options for end users to reach the content and experience the AR enhancements.

In this white paper we make the case that providers of Augmented Reality tools will benefit from a close association with content management systems for print and publishing, and that successful printers and content publishers will integrate the use of Augmented Reality, in a healthy end-to-end ecosystem.

\section{Past, present and future}

In the past, print media and the publishers of content to paper were the "kings" of content. Private and public communications of all kinds were made possible using ink on paper.

We are in a transitional time. Today, content owners are in the process of moving from print to digital media for reaching their target audiences. The print-on-paper and the digital domains are both providing content to users. Content is being broadcast and, at the same time, completely personalized. Increasingly, new media versions of content, designed from the beginning for use in the digital world, are commanding equal or more resources than the print equivalents.

In the information cycle, we are on the left-hand side of Fig. 1. When we complete the cycle this diagram shows, content will be available to users in print, on the web, on tablets and using AR-enabled devices of all types without effort on the parts of the publishers or the users.

Our vision of the future is that any printed material, from a poster, a sign or a package to a printed page in a newspaper, magazine or book, can provide value when combined with a camera, algorithms

\footnotetext{
*Address for correspondence: Christine Perey, PEREY Research \& Consulting, Route de Chernex 2B, 1820 Montreux, Switzerland. E-mail: cperey@perey.com.
} 


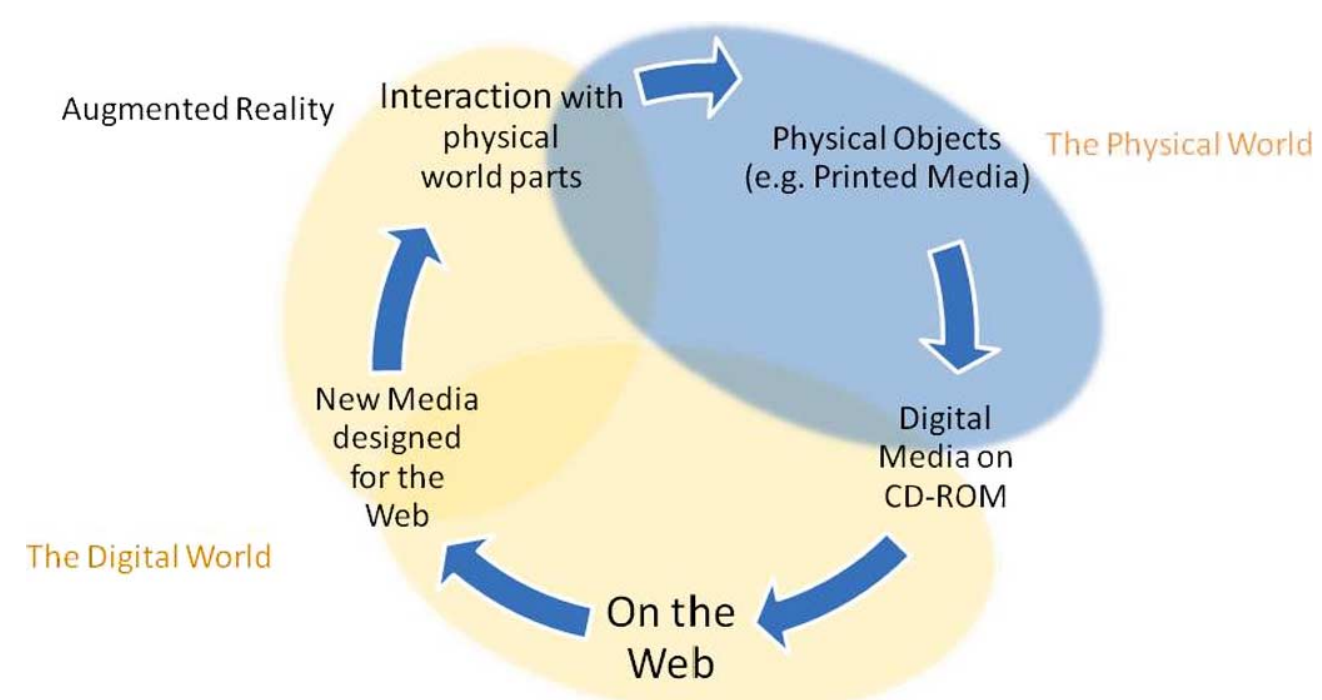

Fig. 1. AR continues what the Internet began. Source: PEREY Research \& Consulting. (Colors are visible in the online version of the article; http://dx.doi.org/10.3233/ISU-2011-0625.)

that detect the content of the page, and platforms that retrieve associated digital data. The combination of AR systems with print will provide value beyond what is possible with either print-only or digital-only content.

In fact, due to the fact that planar (2D) objects are much easier for visual recognition than 3D objects, print will be used more widely as a suitable "trigger" for AR than natural or other man-made objects that do not have a stationary position on the globe. Print will remain in use if for nothing more than to disambiguate and accelerate routine searches on real world objects by AR applications. Further, AR can be systematically used for sight and hearing impaired people, effectively "translating" from one communication medium to another.

\section{Made-for-digital print materials}

Today, when a company prints a URL, a QR code or any type of visual "marker" on their materials, they are communicating with audiences that, at the time of printing, there is supplementary digital material available to go along with the printed object. When the URL appears, the user is being encouraged to go to an Internet-connected device, to enter the URL and to consult the made-for-digital content. When a QR code appears, the user is being instructed to take a photograph of the code using a digital (usually mobile) QR Reader application. The QR reader then transmits the code's unique features to a server on the Internet that compares the user's code features with all the codes in its database and, usually, points the user's browser to a Web page. The Web page can contain any media designated by the publisher and printer of the QR code.

In AR, before natural feature recognition (see sidebar) was widely available, content was triggered by a fiduciary marker or "fiducial". ${ }^{1}$ The camera detects the fiducial, software extracts the most unique "features" of the marker, these are matched to previously designated features stored in a database, in

\footnotetext{
${ }^{1}$ Fiduciary marker, http://en.wikipedia.org/wiki/Fiduciary_marker.
} 


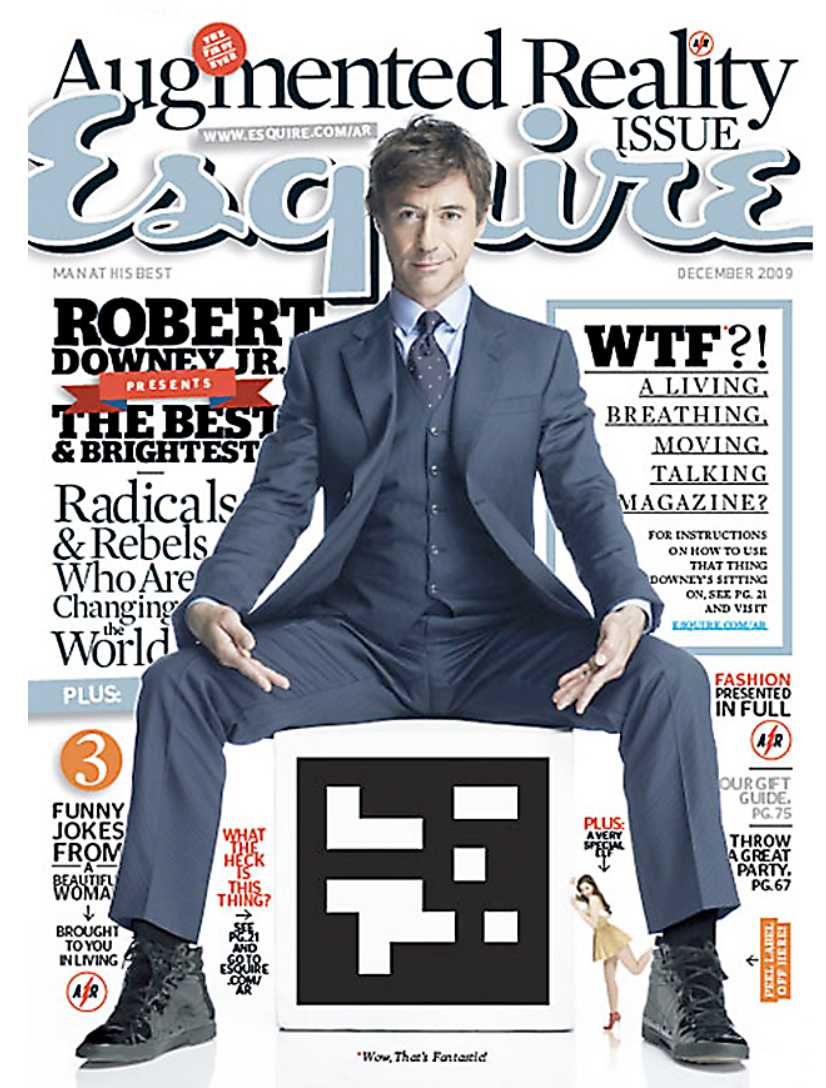

Fig. 2. AR-enhanced experiences with print based on fiducials. (Colors are visible in the online version of the article; http://dx.doi.org/10.3233/ISU-2011-0625.)

a similar fashion to QR codes, and an application sends a digital file (or an "object") to the user's terminal. ${ }^{2}$ The digital file associated with the fiducial in advance is made to appear in real time and in alignment with the marker. The object is visible and sometimes can be interactively explored, in the place of a browser opening a URL to a static Web page. The digital file associated with an AR fiducial on a business card could, for example, be a photo of the person whose name is on the business card.

The fiducial system is currently used in numerous Augmented Reality magazine projects, such as the Esquire December 2009 issue (Barbarian Group using ARToolkitPro) as shown in Fig. 2.

\section{Extending the value and "life" of existing print media}

While the trend towards digital publishing (on the Web and via custom applications) opens innumerable doors for content publishers, audiences and other stake holders in society for low cost and rapid content distribution, there remains a great wealth of content that was originally produced using ink on paper and is only found in hard copy. Reuse or extending the utility of existing print media is an at-

\footnotetext{
${ }^{2}$ What makes the best fiducial?, http://www.vs.inf.ethz.ch/edu/SS2005/DS/papers/ar/owen-bestfiducial.pdf.
} 


\section{What is feature recognition?}

Feature Recognition is a type of Pattern Recognition. Features can be detected by sensors: a camera, a microphone, an accelerometer, etc. Algorithms can extract unique auditory "features" from voices (voice recognition). Specifically visual feature recognition is a method of capturing the visual content of an image or scene for purpose of indexing and retrieval 1.

\section{How can I use it with AR?}

With 2D visual feature recognition, a publisher can elect to enrich all or only a portion of the print materials. A user can be instructed to scan their print materials for interactive areas. These areas may be indicated to the reader with a logo or generic symbol, something which communicates "there's AR here" although, in and of itself, the logo or symbol is not the element which is recognized by the software. The user can also be instructed to point at headlines or text in a specific format.

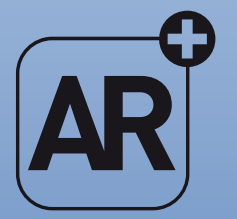

For purposes of creating an AR experience based on a printed page, visual feature recognition must be done not only on a single image , a photo. This is what is used for visual search connected with QR codes and image recognition which brings up a Web page. AR relies on a live video stream (sequence of images) and recognition is performed in real time so that an additional, digital objet is seamlessly integrated with the physical world, if and when the physical world object or the point of view changes.

1. Reference: Image Recognition and Classification: Algorithms, Systems, and Applications by BahramJavidi Publisher: Marcel Dekker, Inc. 2001

tractive value proposition to explore with AR. And, this begins to address the challenge of there being insufficient content to supply to diverse audiences with AR-enabled devices.

Many publishers and content curators have scanned existing print media and the "features" of the letters and pages are rapidly and reliably indexed. Typically, such material is converted to alphanumeric characters and made available in PDF or web pages. Google Books ${ }^{3}$ in particular the Library Project, is an example of the investment one company has made to preserving the images of printed pages while, at the same time, ensuring the content is available in both digital and ink-on-paper formats.

With most of the widely-available AR technologies, a Webcam or mobile cameras and natural feature recognition software with image databases, existing content is "extended" without the need to "insert" or add any special code or markers.

A recent example of how compelling the results can be is the WordLens application for iPhone, published in December 2010 by Quest Visual. ${ }^{4}$ The first release of this mobile AR translation application recognizes words in Spanish and overlays the translated text in English on the user's screen and the

\footnotetext{
${ }^{3}$ Google Books, http://books.google.com/intl/en/googlebooks/about.html.

${ }^{4}$ Quest Visual, http://www.questvisual.com.
} 
reverse (English to Spanish). It is not designed for reading books and does not work in all situations or have support for all languages, but it uses true visual feature recognition-based AR to solve a very real problem experienced by many people. Currently WordLens is a "stand alone" application, which does not require network access to work.

\section{Making AR with print media a reality}

Once the marker or print content or area selected, creating AR with fiducials, a logo or symbol printed into a page or, alternatively, using $2 \mathrm{D}$ visual feature recognition on the printed object itself, involves:

(1) "training" the algorithm to recognize key features within the original content or marker,

(2) creating, usually with 3D software, new, digital content which in some way adds value or "goes with" the existing or new materials, and

(3) connecting the results of \#1, above, the features associated with the "legacy" media (or the madefor-digital page) and the results of \#2, the digital media, with one another.

When the user has access to the publisher-prepared software and databases, visual detection systems need to extract the relevant features from the real world and track these in real time. Finally, the user's device needs to display the digital object in a fashion that is "registered" with the target.

Once these backend and user-enabling systems are in place, the content owner (or its agent) can unlock new revenue streams without cannabilization of the print's inherent business value. Before we get to the point of drawing attention to the combined print-and-digital AR experience, it is important to examine more closely each of the above steps. This workflow is illustrated in Fig. 3.

\subsection{Visual recognition technology}

We briefly introduced the concepts above, but it is important for the printer and publisher to have more background on the potential and shortfalls of this field. Visual recognition is not a new field of research. It has roots in the Artificial Intelligence sector of the mid-1980s, yielding commonplace tools such as Optical Character Recognition (OCR) and it has continued to improve. It is important for many fields such as security, manufacturing, military and medicine. Robotics is one of the fields that are currently driving rapid improvements in real time $3 \mathrm{D}$ visual recognition.

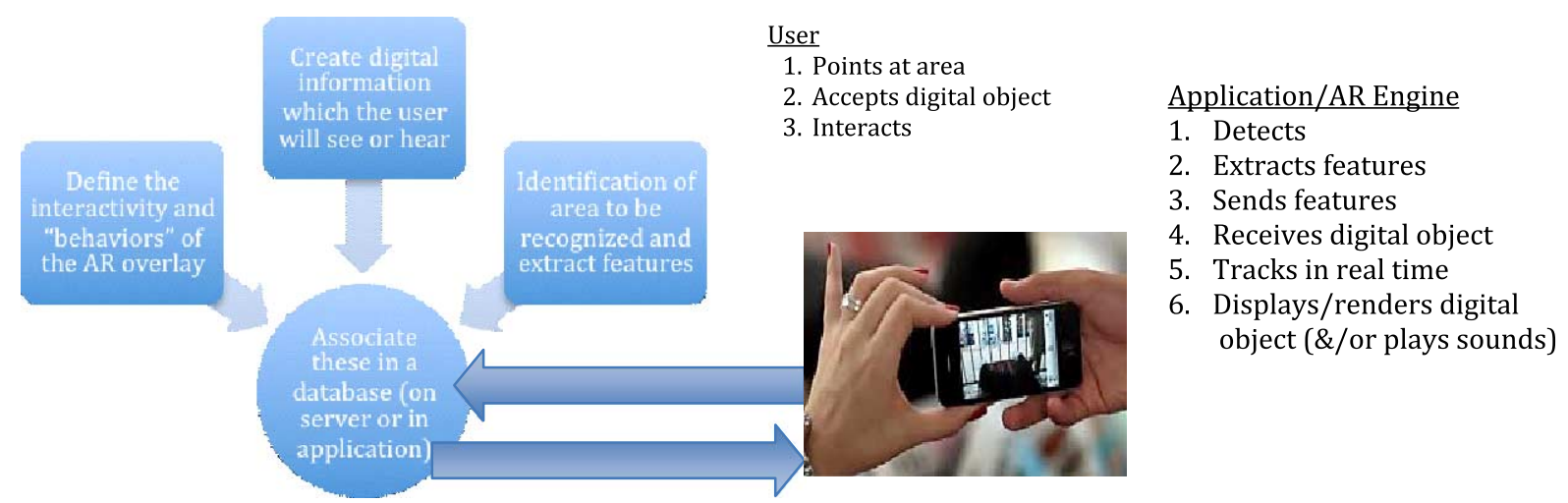

Fig. 3. Workflow diagram of making AR with print media. (Colors are visible in the online version of the article; http://dx.doi. org/10.3233/ISU-2011-0625.) 
The accuracy and speed of recognition systems are getting better but these still do not match the human eye and brain for many applications. Increasing the number and types of visual sensors, and combining geo-position with visual sensing information (cameras) help to alleviate obstacles such as variation due to shadow/lighting effects. To recognize images on deformed surfaces, such as an image that is across the fold of a map or the seam of a book, requires extra processing. To address these real world challenges, visual recognition continues to receive significant investment from commercial companies as well as from research institutes and government agencies.

As indicated in the workflow, for 2D visual recognition and tracking, the technologies used most for AR with print scenarios, the reference image (or many different versions of the same target area) must first be uploaded, the features automatically extracted and these stored in a database.

\subsection{Creating complementary materials}

A typical user does not want to simply look at their screen to see the same content which is on the printed package or page. To be attractive and motivate people to repeat their AR experiences, and even to talk about them with others, the digital content needs to truly add value. Precisely the type of value depends on the target user.

In educational materials, for example, interactivity makes learning more memorable. The use of mobile applications, with and without $\mathrm{AR}$, is shown to enrich the learning of mathematics ${ }^{5}$ and history. ${ }^{6}$

The value may be in the form of recent updates. For example, pointing an AR news application at a headline could bring up an object showing the topic of the headline in a video stream. A graphic placed within a journal article in a scientific publication could trigger the retrieval of a 3D model of the data set.

Creating complex, 3D complementary materials can be time consuming and costly, reducing, in some cases, the financial viability of an AR project. When the purpose of the complementary material is to drives sales of a new product, in a print advertising, for example, the cost of the $3 \mathrm{D}$ content development may be offset by increased revenues from product sales. When margins for a printed material are low, publishers are reluctant to invest in 3D design.

\section{AR content publishing}

In order to gracefully add the AR version of existing and future content to a publisher's readily available (merchandisable) assets, there must be digital content that is stored and managed in a Content Management System (CMS). The CMS should store both the digital objects and the features of the indexed print content. When accessed with AR-capable devices interacting with the printed media made-for-digital or pre-existing - a user has the complete AR (combined print + digital) experience.

A number of barriers exist in this phase of the production and delivery of good AR with print.

Currently, AR content management systems are separate and distinct from print CMS systems. Existing AR platforms require that the indexed print media images (files) be "ingested" into the AR content publishing system. Changes in the media files need to be propagated to all the users and this does not scale well if the data is stored locally to the user.

\footnotetext{
${ }^{5}$ Math4Mobile, http://www.math4mobile.com/.

${ }^{6}$ Learning History with Augmented Reality by Cameron Paterson, October 17, 2010, http://learningshore.edublogs.org/ 2010/10/17/learning-history-with-augmented-reality/.
} 

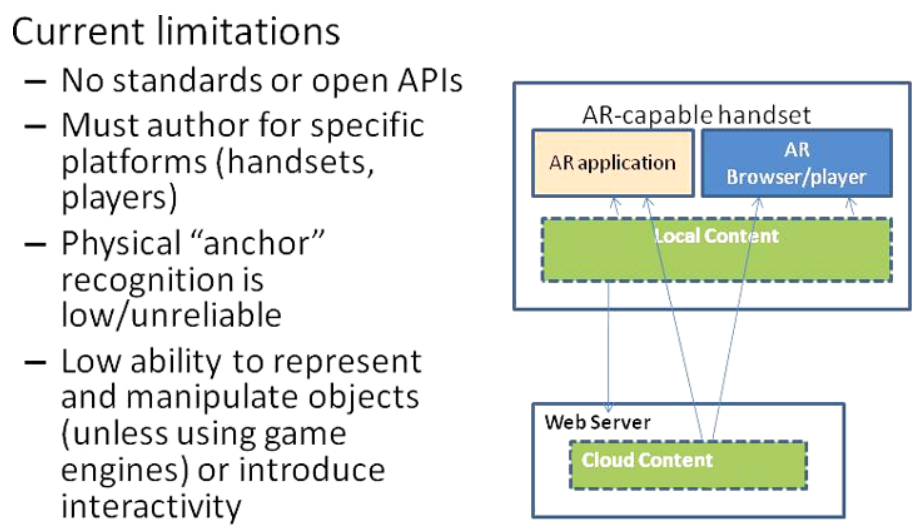

Fig. 4. AR authoring has limitations and tradeoffs. (Colors are visible in the online version of the article; http://dx.doi.org/ 10.3233/ISU-2011-0625.)

Further, due to the unique requirements of different end user devices (e.g., desktop, smartphones, tablets, etc.), entirely different versions of the digital objects may have to be stored in different (separate) databases and provided separately to users. This gets even more fragmented than just a question of whether the user is mobile or stationary. An iPhone user will install a different WordLens application than the user of an Android device (actually, WordLens is not available for Android users).

We also have an inherent conflict between the need to provide content quickly, hence to store as much of the content locally to the user's device, and for the publisher to be able to offer updates via a network. Further, a system must be able to control the use/reuse by users with different agreements with the publisher. Today when the user presents printed matter to a webcam on a PC, many of the objects that are generated in response to a trigger (a product packaging or a printed code, a digital "watch" or a pair of glasses) are stored locally, on the user's device.

These and other limitations are noted in Fig. 4. As the block diagram illustrates, publishing platforms should support having content stored on the device and, also be distributed across the client and server/host for performance without loss of control. In the future, the publishing of AR content must be sufficiently flexible to represent and manipulate objects/support interactivity while, at the same time, offer customized experiences for the different end user platforms.

These are a few of the challenges facing the successful creation of a strong AR-for-print segment.

\section{Collaboration towards the future of $A R$ with print}

In order to overcome challenges such as those described above, the existing ePublishing DRM/CMS system providers for the print and publishing industry (e.g., Adobe, Mark Logic, North Plains Systems, Innodata Isogen, Connotate and Aptara) and the AR platform providers (Total Immersion, metaio, AR Toolworks among others) need to collaborate to simplify the integration of their systems and reduce the barriers to entry for content publishers.

This collaboration will require investments on the part of the:

- AR technology providers,

- those companies providing solutions to publishers and printers, and

- content providers/publishers themselves. 
Investments will take the form of:

- developing open APIs which support the integration of content from visual recognition into an existing publisher's CMS, and/or the reverse,

- tools and workflows which simplify and accelerate the production of 3D objects of suitable interest (with interactivity) and conforming to the needs of a variety of end user devices,

- testing new technologies with existing content as well as with new made-for-digital print materials, and

- educating the marketplace of readers and consumers of information about the new experiences available to them.

\section{Revenues after investments}

In order to justify the shared investments, the partners in new AR products and services for the print and publishing sector must identify the business models which will best match the user and the supplier of content. Printers may charge publishers for additional (custom design) services which produce the 3D AR experiences. Printers may also be the intermediaries for encoding media that was printed prior to the widespread use of AR with print.

Some of the possible sources of revenues for content publishers include:

- charging users for a mobile "companion" application with AR, either one time, upon download or on a subscription basis (monthly, annual, etc.), as is being explored currently with the eBooks and digital magazine publishing applications,

- advertising supported content, either in the printed media or in the digital content received by the user of the AR application or service,

- transactions within the AR application for goods or services, or for additional "value added content" which is not included in the base (possibly free) application.

\section{Conclusion}

We believe that while significant challenges will need to be overcome, there is great potential for AR to be distributed as a companion product by publishers and the channels which market and sell printed media today. We look forward to receiving your feedback to this paper and to supporting the interchange between the two important contributing communities of print and AR. 